\title{
CIUDADES FRONTERIZAS MÉXICO-ESTADOS UNIDOS
}

\author{
Por \\ Alain Vanneph* \\ y Jean Revel-Mouroz**
}

\begin{abstract}
RESUMEN
La extensa frontera México-Estados Unidos posee una especificidad que la diferencia de otras regiones, la organización del espacio urbano a lo largo de la franja fronteriza compuesto por ciudades "gemelas" ubicadas a ambos lados. Desde un enfoque geográfico, en este artículo se analizan diversos elementos económicos, demográficos y sociales que caracterizan a cada par de ciudades vecinas como un solo sistema socio-espacial. Se demuestra la asimetría existente, los contrastes en los paisajes urbanos, así como la creciente interdependencia, característica entre los dos países, manifiesta mayormente en las ciudades "gemelas".

La intensidad de la interacción y los intercambios materiales y humanos a través de la línea divisoria llevan a reflexionar sobre la existencia de una frontera dentro de la misma ciudad.
\end{abstract}

\begin{abstract}
The great length of the Mexico-United States border is responsible for a specific characteristic which makes it different from regions: urban space organized, along the border fringe, into pairs of "twin cities" situated across from each other on opposite sides of the frontier. Taking a geographical point of view, this document analyzes a variety of elements: economic, demographic, and social, characterizing each pair of neighbor cities as a simple socio-geographic system. The existing asymmetry and contrasts in urban landscapes are demonstrated, together with the growing interdependence, which characteristic in both countries is manifested principally in the "twin cities."

The intensity of the interaction and the exchange of people and goods across the dividing line are thought-provoking regard to the existence of an international border inside the city itself.
\end{abstract}

* Maestro de conferencias en París X, investigador del CREDAL, París.

** Director de investigaciones en el CNRS-CREDAL, París.

N. del E. Artículo traducido del francés por Rafael Arriaga Martínez. 
Por sus paisajes, su longitud, la violencia de sus contrastes y la magnitud de los fenómenos migratorios, la frontera México-Estados Unidos constituye un verdadero laboratorio geográfico natural.

Entre todos los componentes que diseñan la especificidad, la importancia y el interés de esta región fronteriza, la originalidad de la organización del espacio, particularmente a través de la configuración de una red urbana de "ciudades gemelas" (una quincena de parejas urbanas que van desde el tamaño de una simple ciudad hasta la de una metrópoli), desarrolladas a ambos lados de la frontera, constituyen uno de los campos de estudio privilegiados del enfoque geografico. En la gestación de este sistema socio-espacial original, las ciudades constituyen por su importancia cuantitativa y por la naturaleza misma de su dispositivo lineal y "gemelar", un paso decisivo en tanto que nudos e interfases de flujos e interacciones múltiples que transforman poco a poco un doble espacio modular en un espacio reticular único que banaliza y aun tiende a borrar la fractura política. Dicha transformación se opera a todos los niveles, pero sobre todo dentro de un mismo espacio urbano.

El propósito de este árticulo, es el de sentar las bases de una reflexión sobre esta evolución transfronteriza, a través de un doble enfoque : ciudad $y$ frontera, $y$ frontera dentro de la misma ciudad.

\section{CIUDAD Y FRONTERA: LA ORIGINALIDAD DE UNA RED URBANA, DEL FRONTERIZO AL TRANSFRONTERIZO}

\section{Desde las ciudades "frontera" hasta la ciudades "Boundary"}

Después de la derrota militar mexicana frente a los Estados Unidos, inmediatamente después de haber quedado trazada una nueva frontera internacional, en 1848 (corregida en 1853) las regiones de la frontera actual se caracterizan por su marginalidad, por su distancia con respecto al centro político y económico de la nueva España. Sin yacimientos mineros que hubiesen podido paliar dicha distancia, la ocupación del espacio por los españoles, reducida a unos cuantos núcleos dispersos, guamiciones militares (presidios) o misiones, constituyen, al lado de las localidades de los pueblos-indios, excepciones en el corazón de un espacio salvaje, olvidado y desértico.

Pocas huellas materiales auténticas quedan pues de ese pasado, incluso en los centros comerciales de algunos embriones urbanos de esa época. Es dentro de una extensión fundamentalmente pionera (frontier) que se opera, a partir de la segunda mitad del siglo XIX, la importancia de los dos lados de esta frontera política (boundary) de casi $3200 \mathrm{~km}$; los ejes ferroviarios 
norte-sur y sus puestos fronterizos tirarán, a lo largo de la primera mitad del siglo Xx, las bases de un crecimiento urbano que vigorizará la creación de distritos de riego y la actividad de comercios y servicios.

La gran originalidad de estas ciudades, de acuerdo con este medio ambiente pionero, es su crecimiento rápido, su carácter de ciudad hongo. En 1900, ninguna de las ciudades de la frontera llegaban a los 20000 habitantes (San Diego tenía 17700 habitantes; El Paso 15900 habitantes; Laredo 13400 habitantes; Ciudad Juárez 8200 habitantes; Mexicali no existía aún y Tijuana era un pueblo de 242 habitantes), mientras que en 1994, tres de estas mismas ciudades llegan al millón de habitantes; en su "hinterland" marcado por la hostilidad de las planicies desérticas que se extienden por todas partes a lo largo de la frontera, desde el Atlántico hasta el Pacífico, la población rural es poca; aun después de la creación de los distritos de riego del Colorado y del Río Bravo, la población agrícola se concentra en las localidades de tamaño urbano. A esta primera originalidad se agrega, en la víspera del siglo $\mathrm{XX}$, particularidades específicas de la huella de una frontera política entre dos estados naciones.

La barrera entre las dos naciones se impermeabiliza al mismo ritmo que resurgen los nacionalismos y en la medida en que esos márgenes desérticos cobran importancia de manera espontánea o estratégica. La línea fronteriza, durante mucho tiempo teórica, se materializa en una verdadera barrera que corta en seco los "hinterlands" difusos de los centros urbanos pioneros.

Este proceso progresivo de cierre, ha inducido desde entonces dos evoluciones urbanas paralelas. Esto implica también una condensación de flujos de intercambio en algunos puntos de paso autorizados y acondicionados; es decir, al este a través de los arroyos o los puentes del Río Grande. Se instaura pues, una "bifurcación" de la evolución, de tal manera que, a partir de una situación idéntica de arranque, se induce poco a poco una gran asimetría entre las naciones, entre las regiones y entre las ciudades.

On the one hand, US and mexican cities are the product of their own national cultures; on the other hand they share a strategic location with their neighbouring urban settlements and increasingly are subject to social, economic, cultural, and even political forces that penetrates the internacional border (cfr. L. Herzog).*

\footnotetext{
* N. del E. Por una parte, las ciudades estadounidenses y mexicanas son el producto de sus propias culturas nacionales; pero, por otra parte, comparten una ubicación estratégica con los asentamientos urbanos vecinos y "crecientemente" están sujetos a fuerzas sociales, económicas, culturales e inclusỏ políticas que penetran la frontera internacional.
} 
La asimetría urbana se inscribe en primer lugar dentro de formas de distribución en el espacio. De acuerdo con los mecanismos clásicos (según los esquemas de Christaller, de Reilly...), las ciudades estadunidenses se desarrollan más en sitios distantes de la frontera, para ampliar al máximo su influencia. Esto se puede observar en la expansión metropolitana de Los Ángeles o Houston, luego de Phoenix, lejos de la frontera política, y en las urbes intermedias de centros secundarios como San Antonio, Tucson o San Diego. El aislamiento justifica la excepción de El Paso.

Por el contrario, en México, se opera un verdadero desdoblamiento entre las capitales de los estados fronterizos (Ciudad Victoria, Monterrey, Saltillo, Chihuahua, Hermosillo) y ciudades fronterizas que a veces las superan en población y peso económico (Nuevo Laredo, Reynosa y Matamoros en Tamaulipas, Ciudad Juárez en Chihuahua). Baja California es la excepción: Mexicali, capital del estado, se ha fundado en la frontera misma, en el corazón de ese gran distrito de riego que es el valle del Río Colorado.

La vocación fronteriza de Tijuana, Mexicali, Ciudad Juárez, Nuevo Laredo, Reynosa y Matamoros, ha hecho que éstas crezcan en forma de embudo, pegadas a lo largo de la línea internacional.

Uno de los mayores elementos de asimetría que le han dado un cierto dinamismo al lado estadunidense es la actividad militar. Mientras que hoy en día, los mexicanos marcan su presencia en la frontera con unos cuantos batallones militares (viejos herederos de los "presidios"), los estadunidenses aprovechan los espacios desérticos disponibles en Texas, Nuevo México y Arizona al transformar en bases y sitios de pruebas la línea de fuertes creados después de 1848 a lo largo del Río Grande y del eje de comunicación este-oeste, hasta California. Asimismo, en lo que respecta a la bahía de San Diego, que ha hecho de esta ciudad una gran base naval/aéreo-naval del Pacífico, el ejército, con sus militares, su personal civil (entre unos y otros, varios cientos de miles) y sus instalaciones, marcan de manera permanente la actividad y el paisaje de las ciudades como El Paso y San Diego, y a veces, sobre todo durante los periodos de conflicto (segunda guerra mundial, Corea, Vietnam) algunas bases secundarias como la de Laredo, McAllen, entre otras.

Más concretamente aún, la asimetría se inscribe dentro de los contrastes de los paisajes urbanos. Las "ciudades gemelas" son falsas gemelas (cfr. A. Venneph y J. Revel-Mouroz), son lugares de diferencia, en relación con otras localidades nacionales no fronterizas y en relación de una ciudad "gemela" con respecto a la otra. Ciudad latina y del tercer mundo por una parte, frente a la ciudad anglosajona y estadunidense. Es de imaginarse hasta dónde la oposición sistemática de todos los caracteres de estas dos 
realidades urbanas, puestas frente a frente, a cada lado de una simple alambrada fronteriza o de un estrecho río, se traduce en contrastes realmente caricaturescos. Y por lo tanto, si del cierre de la frontera ha surgido - por efecto de filtro- una urbanización doblada, de la misma manera, de esta diferencia de potencial y de esta proximidad han surgido complementariedades e interacciones; nacidas de la frontera y desarrolladas por la frontera, éstas terminan finalmente por cuestionar la frontera misma: "Rio Grande does not separate people...Along the entire border, towns are twins and siamese twins in some cases, for many interconnecting communications" (cfr. Williams, 1968).*

Entre 1940 y 1990 , la población de los condados fronterizos estadunidenses ha sido multiplicada por 5.5 (pasando de 952000 a 5197 000 habitantes) y la de los municipios fronterizos mexicanos por 9.6 (pasando de 395000 a 3810000 ). Dicha diferencia de crecimiento ha permitido a la mayor parte de las ciudades mexicanas alcanzar y rebasar a sus homólogas estadunidenses, por lo menos en habitantes; las diferencias de estructuras de edad y de ingreso per cápita continúa concediéndoles a las ciudades estadunidenses un potencial de trabajo y un peso economico y un poder de compra muy superior a sus homólogas mexicanas, excepcion hecha de las parejas caricaturescas de tipo Mexicali/Caléxico, capital del estado de Baja California la primera, y ciudad de servicios la segunda.

Desde el punto de vista estrictamente demográfico, sólo la ciudad de San Diego sigue siendo netamente más importante que su gemela Tijuana, a pesar del récord de crecimiento que registra esta última.

\section{Hacia un nuevo encuentro}

La interdependencia creciente, característica de las relaciones entre México y Estados Unidos, llega a su apogeo entre las ciudades gemelas fronterizas, de manera paralela a la multiplicación de intercambios entre uno y otro lado de la línea internacional. La oposición deja lugar a la complementariedad. Más que su diversidad y su volumen, es quizás la naturaleza y el carácter sistemático de dichas relaciones lo que se debe subrayar.

Relaciones de ida y vuelta, reacciones de reciprocidad, retroacciones que inducen al desarrollo de un verdadero sistema urbano binacional y aun

\footnotetext{
* N.del E."El Río Grande no separa a la gente... A lo largo de la frontera, las ciudades son gemelas o en algunos casos siamesas con respecto a las comunicaciones que se interconectan".
} 
transnacional: "urbanisation has become a boundary transcending process"* (cfr. Lawrence Herzog, 1990:249).

Un juego complejo de contradicciones no antagónicas (cfr. Raúl Fernández) tiende a transformar las parejas en conurbaciones, y a San Diego-Tijuana o El Paso-Ciudad Juárez en metrópolis.

$\mathrm{Al}$ interior de la constitución de este modelo, los flujos tradicionales de mercancías (por ejemplo 1000 camiones diarios en la aduana de Mesa de Otay en Tijuana), y sus corolarios aduanales y fiscales han contado, es cierto, pero dentro de un desarrollo urbano propio a cada lado de la ruptura de la carga, más, en términos de complementariedad que de interactividad - como se puede observar, por ejemplo, en los dos Nogales a partir del transporte de ganado y de frutas y legumbres que México exporta.

Las cosas se presentan de otra manera en lo que se refiere a intercambios migratorios, cuyo rol ha sido importante para los dos lados. Por el lado estadunidense tenemos la implantación, temporal o definitiva, de varios millones de mexicanos - los braceros y sus descendientes-en el suroeste de Estados Unidos, particularmente en las ciudades fronterizas. Aunque también - y es un primer ejemplo de retroacción del lado mexicano- la recepción de repatriados en las ciudades mexicanas y sobre todo, el rol de las "salas de espera" (Staging Area) que juegan esas mismas ciudades ante los candidatos a la emigración, han alimentado a estas ciudades con una población flotante importante: $7 \%$ de la población de Mexicali, por ejemplo, 6 \% de la de Baja California (cfr, Gabriel Estrella, 1992).

Dicha población, aunque también parcialmente estabilizada, se renueva constantemente en función de los plazos que impone tanto el "filtro" fronterizo como los regresos sucesivos a las ciudades fronterizas que se encuentran de paso entre el lugar de origen y el lugar de destino en los Estados Unidos. Además de ello, están las filiales especializadas en el tráfico de indocumentados que poco a poco han constituido una de las fuentes de un crecimiento urbano multiplicado gracias a la gran fecundidad de las madres mexicanas.

Entre los traslados de migrantes, de turistas y de compradores de vecindad, las ciudades de la frontera se han convertido en lugares de paso incesante. Tijuana se jacta de tener el flujo más importante del mundo. Las estadísticas más fiables, las que miden las corrientes sur-norte, es decir, en dirección hacia Estados Unidos, confirman esta visión de ciudades frontera que funcionan con puertas dobles (más de 200 millones de cruces anuales sur-norte).

\footnotetext{
* N. del E. "La urbanización se ha convertido en un proceso que trasciende la frontera".
} 
Cuadro 1. Crecimiento de la población en los principales municipios, condados y ciudades de la frontera. (1940-1990).

\begin{tabular}{|c|c|c|c|c|c|c|}
\hline \multirow[t]{2}{*}{$\mathrm{ENC}=$} & \multicolumn{6}{|c|}{ Municipios (m), ciudades (C) y condados (c) de México y Estados Unidos. } \\
\hline & 1940 & 1950 & 1960 & 1970 & 1980 & 1990 \\
\hline Tijuana (m) & 21977 & 65364 & 165690 & 340583 & 461257 & 747381 \\
\hline Tijuana (C) & 16486 & 59952 & 152374 & 277306 & 429500 & 698752 \\
\hline San Diego (c) & 289348 & 556808 & 1033011 & 1357854 & 1861846 & 2498016 \\
\hline San Diego (C) & 203341 & 334387 & 573224 & 696769 & 875538 & 1110549 \\
\hline Mexicali (m) & 44399 & 124362 & 281333 & 396324 & 510664 & 601938 \\
\hline Mexicali (C) & 18775 & 64609 & 174540 & 263498 & 341600 & 438377 \\
\hline Imperial (c) & & & 92 & 110 & 109 & 303 \\
\hline Caléxico $(\mathrm{C})$ & 5415 & 6433 & 7992 & 10625 & 14412 & \\
\hline S. L. Río Colorado (m) & 2364 & 13593 & 42134 & 63604 & 92790 & 110530 \\
\hline S. L. Río Colorado (C) & 558 & 4079 & 28545 & 49990 & 76673 & 95461 \\
\hline Yuma (c) & 19326 & 28006 & 46235 & 60827 & 90554 & 106895 \\
\hline Yuma (C) & 5325 & 9145 & 23974 & 29007 & 42433 & 54000 \\
\hline Nogales (m) & 15422 & 26016 & 39812 & 53494 & 68076 & 107936 \\
\hline Nogales (C) & 13866 & 24478 & 37657 & 52108 & 65587 & 105873 \\
\hline Santa Cruz (c) & 9482 & 9344 & 10808 & 13966 & 20459 & \\
\hline Nogales (C) & 5135 & 6153 & 7286 & 8946 & 15683 & 19500 \\
\hline Juárez (m) & 55024 & 131308 & 276995 & 42 & 567 & 797679 \\
\hline Ciudad Juárez (C) & 48881 & 122566 & 262119 & 407370 & 544496 & 789522 \\
\hline El Paso (c) & 131067 & 194968 & 314070 & 359291 & 479899 & 591610 \\
\hline El Paso (C) & 96810 & 130485 & 276687 & 322261 & 425259 & \\
\hline Piedras Negras (m) & 18667 & 31665 & 48 & 8 & 80 & 98185 \\
\hline Piedras Negras (C) & 15663 & 27581 & 44992 & 41033 & 67444 & 96178 \\
\hline Maverick (c) & 10071 & 12292 & 14508 & 18093 & 31398 & \\
\hline Eagle Pass (C) & 6459 & 7267 & 12094 & 15364 & 21407 & \\
\hline Nuevo Laredo (m) & 31502 & 59496 & 3 & 15 & 86 & 219468 \\
\hline Nuevo Laredo (C) & 28872 & 57668 & 92627 & 148867 & 201690 & 218413 \\
\hline Webb (c) & 45916 & 56141 & 64791 & 72859 & 99258 & 133239 \\
\hline Laredo (C) & 39274 & 51510 & 60678 & 69024 & 91449 & 108700 \\
\hline Reynosa (m) & 23137 & 69428 & 134 & & 211 & 282667 \\
\hline Reynosa (C) & 9412 & 34087 & 74140 & 137383 & 194657 & 265663 \\
\hline Hidalgo (c) & 106059 & 160446 & 180904 & 181535 & 283229 & 383545 \\
\hline McAllen (C) & 11877 & 20067 & 32728 & 37636 & 66281 & \\
\hline Matamoros (m) & 54136 & 128347 & 143043 & 186146 & 238840 & 303293 \\
\hline Matamoros (C) & 15699 & 45846 & 92327 & 137749 & 188703 & 266055 \\
\hline Cameron (c) & 83202 & 125170 & 151098 & 140368 & 209727 & 260120 \\
\hline Brownsville (C) & 22063 & 36066 & 48040 & 52522 & 84997 & 94700 \\
\hline
\end{tabular}

Fuente: U.S. Census of Population y Censos generales de población. 
Cuadro 2. Entradas a Estados Unidos, en 1989 (en miles).

\begin{tabular}{|c|c|c|c|c|c|c|}
\hline $\begin{array}{l}\text { Lugar de ingreso } \\
\text { y rango }\end{array}$ & Total & $\begin{array}{c}\text { adas } \\
\%\end{array}$ & Ext & $\%$ & Ciudac & $\begin{array}{r}\mathrm{E}-\mathrm{U} \\
\%\end{array}$ \\
\hline 1 San Diego (Ca.) & 63587 & 4.8 & 34840 & 13.5 & 28747 & 16.9 \\
\hline 2 El Paso (Texas) & 42358 & 9.9 & 24753 & 9.6 & 17605 & 10.4 \\
\hline 3 Laredo (Texas) & 27944 & 6.5 & 19675 & 7.6 & 8269 & 4.9 \\
\hline 4 Calexico (Ca.) & 24912 & 5.8 & 18908 & 7.3 & 6004 & 3.5 \\
\hline 5 Detroit (Michigan) & 21893 & 5.1 & 10796 & 4.2 & 11097 & 6.5 \\
\hline $6 \mathrm{Hid}$ & 176 & 4.1 & 118 & 4.6 & 5812 & 3.4 \\
\hline 7 Nogales (Arizona) & 17499 & 4.1 & 11482 & 4.4 & 6017 & 3.5 \\
\hline 8 Brownsville (Texas) & 14831 & 3.5 & 9909 & 3.8 & 4922 & 2.0 \\
\hline entradas & 429 & 100 & 4 & 100 & 170096 & 100 \\
\hline $\begin{array}{l}\text { ntradas a través } \\
\text { la frontera } \\
\text { lexicana }\end{array}$ & 208748 & 48.7 & 131372 & 50.7 & 73376 & 43.1 \\
\hline
\end{tabular}

Fuente: Servicio de Inmigración y Naturalización de los Estados Unidos. Ingreso de extranjeros y ciudadanos por puertos de entrada. Año fiscal 1989.

Este tipo de fenómenos acumulativos, este sistema de interacción, se refuerzan todavía más con el juego complejo de la industria maquiladora; esa industria ensambladora, sin aduana para la exportación y para la cual el gobierno mexicano creó en 1965 un régimen aduanal y fiscal particular, a lo largo de la frontera. Las fábricas (en electrónica, en equipo para automoviles, de ropa) que de hecho trabajan para el mercado norteamericano, se inscriben directamente dentro de un campo regional de proximidad. Dichas fábricas privilegian la complementariedad localizada en ambos lados de una frontera viva, antes que todo como el límite de un deposito de mano de obra.

Las ciudades mexicanas de la frontera se han transformado así en modelos de ciudades industriales incluidos dentro de un sistema transnacional y transfronterizo, con una aceleración del número de implantaciones y empleos asociados desde 1983. 
Cuadro 3. Localización de maquiladoras (subrayado para la localización fronteriza).

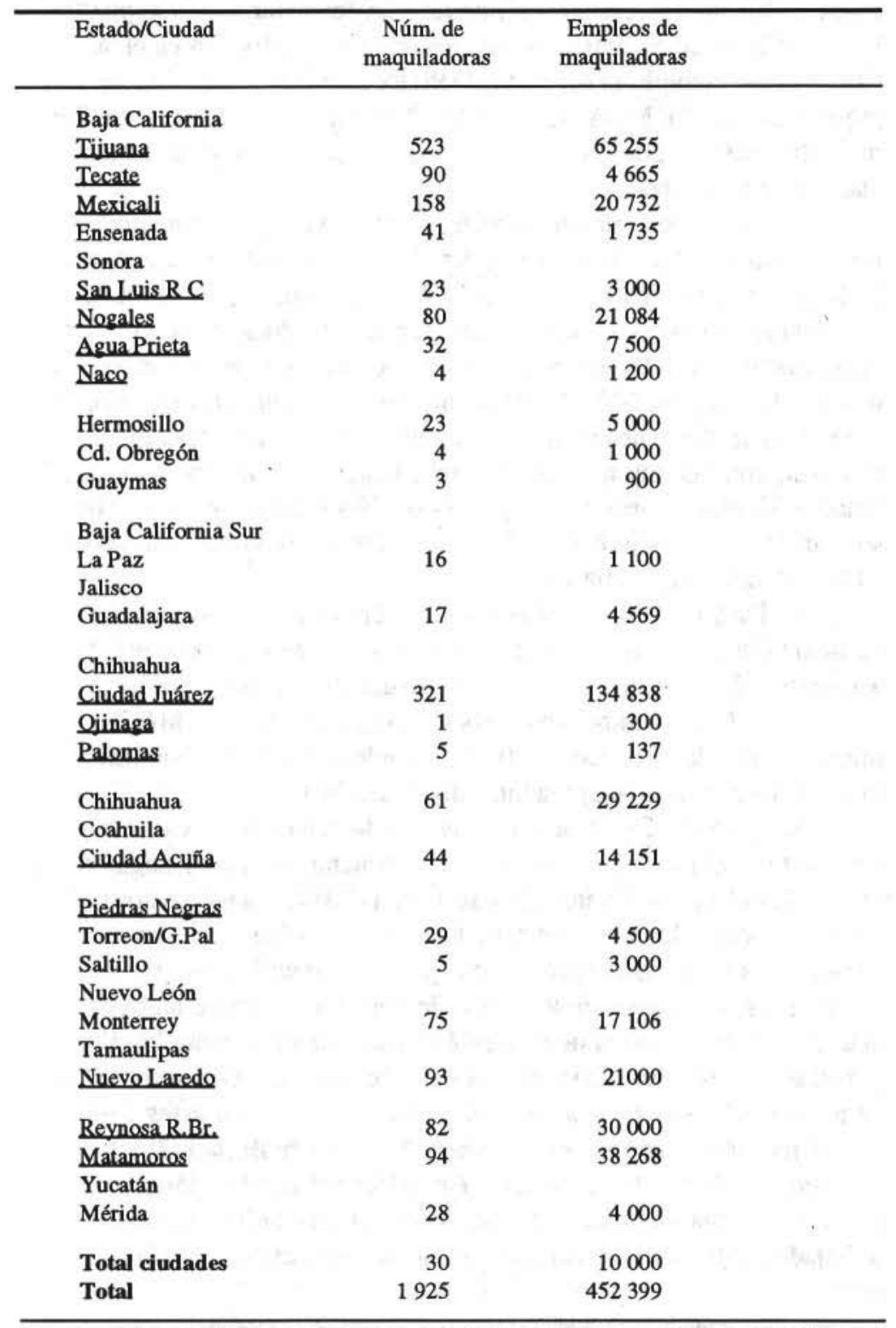

Fuente: “Monthly Scoreboard”, Twin Plant News El Paso, vol 7, núm.1 (1991:68). 
Al fenomeno de ciudad gemela, se sobrepone un fenómeno de "plantas gemelas", que fija en el lado norte - dentro de la ciudad más proxima, cerca de los nudos "carreteros", de las vías ferroviarias y aeropuertoslas actividades complementarias de los montajes realizados en el lado sur. Citemos por ejemplo el lugar de MANITEX en McAllen, o bien las zonas industriales de la Mesa de Otay en Tijuana y San Diego, en donde maquiladoras y "plantas gemelas" japonesas vienen y se pegan a la alambrada fronteriza.

Zonas libres en perímetro cerrado forman así el pendiente que cuelga por el lado estadunidense, alrededor de $20 \mathrm{~km}$ fuera de la aduana, establecida en la vertiente mexicana. El $42 \%$ de las industrias maquiladoras de Tijuana disponen de una "planta gemela" en San Diego. 15\% de la industria de San Diego mantiene relaciones de negocios con una maquiladora y para $60 \%$ de éstas, tal relación implica un reclutamiento específico de dirigentes y gerentes adaptados a esta clase de comunicaciones. Asfi, con las múltiples actividades, ligadas de manera indirecta, los Estados Unidos obtienen de regreso - y ésta sería la primera forma de solidaridad transfronteriza- $80 \%$ de empleos creados (o conservados) por cada 10 empleos maquiladores.

En El Paso-Ciudad Juárez la simbiosis entre ciudades gemelas de gran tamaño es la más avanzada y es en donde la interdependencia es la menos asimétrica, debido a la conjunción del dinamismo de los grupos mexicanos locales y de la solidaridad de los socios locales de El Paso. México es "la mina de oro del El Paso"; 20000 empleos están ligados, directa o indirectamente, a las maquiladoras de Chihuahua.

A este primer acoplamiento, a nivel de la mano de obra -el que, por otra parte, podriamos oponer a ese acoplamiento antiguo y negativo que resulto de la localización inicial—, se agrega el de los movimientos cotidianos que realizan los dirigentes estadunidenses o asiáticos de las maquiladoras, que aprovechan la proximidad para residir en Estados Unidos.

Ya no se trata pues, únicamente de coexistencia entre ambas partes, sino de una cierta forma de solidaridad transfronteriza entre las ciudades gemelas y, con las interconexiones industriales, del primer encadenamiento del futuro: "The maquilas help the two countries overcome differences by doing business together" * (Angélica Becker, 1990).

Aun cuando puede haber una red transfronteriza industrial de "subcontratacion", el mayor impacto del empleo se queda en México, en donde los trabajadores de las maquiladoras representan, en general, entre una quinta

\footnotetext{
* N. del E."Las maquiladoras ayudan a los dos países a superar diferencias al hacer negocios en conjunto".
} 
Cuadro 4. Población activa en principales ciudades fronterizas, 1990.

\begin{tabular}{lrrrrrrr}
\hline Ciudad & $\begin{array}{c}\text { Población } \\
\text { ocupada }\end{array}$ & $\begin{array}{c}\text { A pob. } \\
\text { activa }\end{array}$ & $\begin{array}{c}\text { B pob. } \\
\text { secundaria }\end{array}$ & $\begin{array}{c}\text { C. empl. } \\
\text { terciaria }\end{array}$ & D.pob. & B/A\% & C/A\% \\
\hline Mexicali & 438377 & 148061 & 42599 & 20566 & 88517 & 28,8 & 13,9 \\
Tijuana & 698752 & 245731 & 93265 & 57296 & 140008 & 38,0 & 23,3 \\
Nogales & 105873 & 38286 & 17994 & 17909 & 19064 & 47,0 & 46,8 \\
Ciudad Juárez & 789522 & 280160 & 138161 & 121004 & 126520 & 49,3 & 43,2 \\
Nuevo Laredo & 218413 & 69429 & 23195 & 15608 & 42082 & 33,4 & 22,5 \\
Reynosa & 265663 & 86236 & 36182 & 24980 & 44920 & 42,0 & 29,0 \\
Matamoros & 266055 & 95101 & 46893 & 35977 & 43083 & 49,3 & 37,8 \\
\hline
\end{tabular}

Fuente: Elaborada por los autores con base en: INEGI. Censo de Población, 1990.

(*) INEGI. Estadísticas mensuales de la industria maquiladora. 1990 nota: Dada la diferencia de las fuentes, la relación C/A es indicativa.

Cuadro 5. Salarios pagados por el Departemento de la Defensa y monto de los contratos militares, 1992.

\begin{tabular}{|c|c|c|c|c|}
\hline \multirow[b]{2}{*}{ Condado } & \multicolumn{2}{|c|}{$\begin{array}{l}\text { Salarios pagados por el } \\
\text { epartamento de la Defensa }\end{array}$} & \multicolumn{2}{|c|}{$\begin{array}{l}\text { Monto de los contratos } \\
\text { militares. }\end{array}$} \\
\hline & $\begin{array}{l}\text { Monto } \\
\text { millones \$EUA }\end{array}$ & $\begin{array}{c}\text { Rango } \\
\text { nacional }\end{array}$ & $\begin{array}{c}\text { Monto } \\
\text { millones \$EUA }\end{array}$ & $\begin{array}{l}\text { Rango } \\
\text { nacional }\end{array}$ \\
\hline San Diego & 3670 & 1 ro. & 2480 & 7o. \\
\hline Bexar (San Antonio) & 1722 & 50. & & \\
\hline El Paso & 973 & 11o. & & \\
\hline Condado de Los Ángeles & 804 & 13o. & 10809 & 1ro. \\
\hline
\end{tabular}

Fuente: Economic Research Bureau of the Greater San Diego Chamber of Commerce.

parte y la mitad de la población activa en la ciudades de la frontera, cambio profundo en relación con la hegemonía comercial y terciaria que imperaba aún hace veinte años.

Las industrias de la región conocida como "Sun Belt", y las industrias de "subcontratación" mexicana cuentan con una sensibilidad común con respecto a la coyuntura; de esta manera, $30 \%$ de las empresas de Arizona están en relación con México, $30 \%$ de las exportaciones industriales del estado se efectúan a través de las maquiladoras, y en total, $20 \%$ del abastecimiento de las maquiladoras provienen de la región fronteriza estadunidense (cfr. Silver, 1991). 
Dentro de la coyuntura general del comportamiento de la economía estadunidense y de la demanda, un sector particular resulta afectado con una baja tendencia: el de la defensa. Ahora bien, este sector es uno de los pilares de la economía local de las principales ciudades estadunidenses de los estados de la frontera.

Al final de los años ochenta, las bases de Fort Bliss, White Sands, Holloman, y las industrias bajo contrato (Rockwell, United Technologiques, Martin Marrieta, Honeywell), le inyectaban un millar de dólares por año a la economía regional de El Paso, en donde 150000 personas dependían de la defensa (militares, jubilados, empleados civiles u obreros). El peso de las actividades ligadas al sectormilitar eran de $20 \%$ con respecto al producto de El Paso. En San Diego, aunque este porcentaje no sea más que $16 \%$, de todas maneras es el segundo sector de actividad local después de la industria, con un peso de alrededor de 10 millones de dólares y más de 400000 personas involucradas.

$\mathrm{La}$ actividad de las ciudades mexicanas de la frontera ha estado tradicionalmente ligada al sector militar estadunidense, sobre todo a través de la adquisición de bienes y servicios que esta población realiza (que da vida a una parte del sector noctumo), aunque también a través de la subcontratación industrial, aun dentro del sector high-tech (se dijo durante la guerra del Golfo Pérsico que $10 \%$ de los componentes del misil Tomahawk habían sido fabricados en México).

Si bien la iniciativa de defensa estratégica ha impulsado la actividad de investigación y desarrollo industrial de El Paso, Arizona y Califomia, los cortes en los presupuestos militares realizados desde 1989 le han dado mayor peso tanto a las actividades de alta tecnología civil como a las que están ligadas a la industria maquiladora mexicana.

Cuadro 6. Personal militar y salarios, 1992.

\begin{tabular}{lcccc}
\hline & $\begin{array}{c}\text { San Diego } \\
\text { efectivos }\end{array}$ & $\begin{array}{c}\text { Salarios } \\
\text { (millones) }\end{array}$ & $\begin{array}{c}\text { El Paso } \\
\text { efectivos }\end{array}$ & $\begin{array}{c}\text { Salarios } \\
\text { (millones) }\end{array}$ \\
\hline Militares activos & 133671 & 3647 & & \\
Miembros de familia & 196000 & & & \\
Empleados civiles & 31543 & 573 & & \\
Beneficiarios retiros & 56497 & 862 & & \\
Total & 417711 & 5081 & $133915(1989)$ & $756(1988)$ \\
\hline
\end{tabular}

Fuente: San Diego Economic Bulletin, vol, 41, núm. 6, junio 1993 y Public Affairs Office, Fort Bliss. 
La evolución de los dos lados de la frontera hacia la mundialización y los "flujos tensos", ha desembocado además en una fuerte demanda de telecomunicaciones y en la construcción de redes transfronterizas centradas en las ciudades de la frontera que evolucionan en nudos estratégicos de comunicación.

Un fenomeno típico de retroacción en "rizo" se nutre de esta nueva función que se impone a raíz de las necesidades específicas de tele-gestion de las maquiladoras y que, a su vez, por su existenciamisma, puede facilitar la creación de otras maquilas o industrias de "just-in-time". Éste es el caso de Tijuana, en donde gracias a la red telefónica privada Teléfonos del Noroeste (TELNOR), la ciudad se beneficia de la tasa más alta de digitalización mexicana $-\mathrm{y}$ por ende, de la más alta eficiencia -, $80 \%$ contra $20 \%$ de la media nacional para las grandes ciudades; Baja California llega a alcanzar mejores promedios para sus ciudades pequeñas, como es el caso de Ensenada (90\%) (cfr. Eduardo Barrera Herrera, 1992).

Por todos lados de la frontera mexicana, la necesidad de conexiones internacionales acarrea la multiplicación de servicios complementarios a Teléfonos de México (TELmeX). Cada ciudad fronteriza dispone de un promedio de 300 instalaciones microondas (con o sin autorización oficial). Ciudad Juárez, verdadero telepuerto, "capital mundial de la maquila y del by-pass", dispone de 720.

A todo esto se agrega la telecomunicación directa a través de canales privados vía satélite (como es el caso de las maquiladoras SANYO, KENMEX - Matsushita de Tijuana), y la red especial "red superpuesta" que TELMEX propone en la frontera, así como las comunicaciones locales, celulares, que desde El Paso, por ejemplo, cubre todo Ciudad Juárez.

La noción pues de ciudad "conectada" aparece como primordial para el futuro. Constituye en efecto una ventaja comparativa (en equipamiento y savoir faire) en tanto que elemento de cultura, de modernidad y de apertura, confiriéndole a la región fronteriza, en relación con la proximidad física de los Estados Unidos, de sus equipos y de sus mercados, una cualidad propia que perdurará, aun dentro del cuadro del Tratado de Libre Comercio (TLC).

Entre los otros flujos que alimentan de manera creciente la interdependencia de las ciudades gemelas, figura en buen lugar el turismo. Dicho flujo es importante no sólo por su volumen (Tijuana se jacta de ser la ciudad más visitada del mundo con 65 millones de cruces fronterizos registrados en 1992, aunque sólo el $4 \%$ de los turistas se quedan más de un día) o por su movimiento en doble sentido, sino por la complejidad y la retroacción de las relaciones transfronterizas que se tejen alrededor de esta función y otras actividades locales. 
En ambos lados de la frontera se establece, de hecho, un sistema interactivo, asociando turismo, maquila y comercio de las ciudades gemelas.

Una parte importante del turismo anglosajon hacia México beneficia a las ciudades fronterizas estadunidenses. Esto se puede constatar en San Diego, por donde pasa $25 \%$ de los turistas que recibe Tijuana. Esto también se puede constatar en McAllen, donde los recursos hoteleros ligados a las migraciones invernales de los jubilados del "Manufacturing Belt", hacia el sur, se encuentran reforzados y complementados con el rol de paso hacia Reynosa, ciudad que ofrece nada más 300 cuartos de hotel, en tanto que la ciudad texana de McAllen ofrece 1500 cuartos, Recíprocamente, se observa que $57 \%$ de las personas que visitan Tijuana son de ascendencia mexicana (crf. Nora Bringas, citado en Olivia Ruiz, 1992).

El rol del turismo comercial no es menos complejo. Las compras clásicas de curiosidades y servicios (principalmente reparación y carrocería de autos, y todas las operaciones comerciales relacionadas con el "turismo del vicio"), hechas por los residentes estadunidenses en las ciudades mexicanas, se efectúan prácticamente, mientras que los residentes mexicanos compran en las ciudades estadunidenses. Así, los trabajadores petroleros de Reynosa compran en promedio una vez por semana en McAllen. Según la Secretaría de Comercio y Fomento Industrial (SECOFI), 35\% de los ingresos de Reynosa se gastan en Estados Unidos.

En Arizona se observa la misma proporción; se gasta un $30 \%$ de los salarios correspondientes a las maquilas fronterizas. Es de esta manera que en Tucson se genera un ingreso anual de 28 millones de dólares por concepto de compras hechas por mexicanos, y en la frontera misma (condado de Santa Cruz), 60\% del comercio por menudeo depende del vaivén de los mexicanos (cfr. Pavlakovic s/f). En San Diego, los trabajadores de las maquilas gastan 90 millones de dólares cada año. En nogales, Arizona, $60 \%$ del comercio por menudeo depende también de las compras mexicanas.

Una de las mejores ilustraciones de esta interdependencia, creadora de una verdadera "área de reciprocidad transfronteriza", puede ser la sensibilidad de toda la actividad económica de las ciudades estadunidenses a las variaciones de la paridad del peso en relación al dólar. Así, la devaluación de la moneda mexicana en 1976 , se tradujo en una baja de $25 \%$ en los cruces fronterizos registrados en Texas-Tamaulipas. Las entradas comerciales de McAllen bajaron 1\%, siendo que el año anterior había registrado más de $25 \%$. En sentido inverso, la relación de compra en México provocó una alza de $24 \%$ de las ventas por menudeo registradas en Reynosa, lo que favoreció la implantación de nuevos mercados, mientras que con la segunda gran devaluación, a partir de 1982, se recupera el 
proceso de implantación de los grandes mercados en el lado mexicano. Hasta entonces, los centros comerciales periféricos de las ciudades mexicanas se localizaban de hecho en Estados Unidos. Así, McAllen llega a totalizar 22 grandes superficies comerciales y 1.7 millones de dólares de ventas anuales, lo que lo coloca en volumen, en el noveno lugar entre las ciudades texanas, y en superficie comercial, por habitante, según las autoridades locales (1988), en el segundo lugar a nivel mundial, después de Berlín.

De la misma manera, las ventas de menudeo en San Isidro, localidad fronteriza del condado de San Diego, se demumban en $40 \%$ después de la devaluación de 1976, y en $7.5 \%$ después de 1982. Una diferencia que a su manera aporta la prueba del profundo arraigo de las relaciones y las dependencias casi estructurales de los mexicanos con respecto al otro lado. En El Paso se habla todavía del "temblor de la devaluación de 1982". Ello' están concientes que sin México, sus compradores y sus maquiladoras, El Paso sería en Texas algo así un Midland sin petróleo.

La ciudad estadunidense, gracias a la seguridad por las cuentas en dólares, aparece igualmente como "caja fuerte" y "city" a los ojos de la población-conurbación binacional. En McAllen, hay 15 bancos (para 80000 habitantes dentro de los límites administrativos del municipio). En El Paso, el sector financiero es particularmente activo, debido a los depositos mexicanos en sus bancos (más de 700 millones de dólares en 1988), 20\% de la actividad inmobiliaria y hasta $30 \%$ de las ventas de los centros comerciales dependen de los compradores mexicanos. Se trata de una ciclo financiero que funciona a través de la frontera, con toda una serie de tráficos y reinversiones que van más allá de un simple movimiento altemativo.

Asistimos en consecuencia a una especialización funcional de las dos ciudades, a la manera de los barrios de una misma metrópoli, o a una especialización de barrios en función binacional, en lugar de dos barrios nacionales. Esto se refleja principalmente en los fenómenos de CBD de la ciudad estadunidense que asocia sus empresas locales, comerciales o industriales, a las oficinas anexas y a veces principales de las empresas mexicanas. Así lo demuestran las 5800 empresas hispanas establecidas en McAllen, cuarto lugar en Estados Unidos en lo que respecta a la localización de actividades mexicanas o méxico-americanas.

La ciudad del norte se especializa igualmente en servicios de alta calidad para la clientela local y las elites mexicanas; por ejemplo, los servicios de salud. A la ciudad del sur le corresponderían las atenciones ordinarias, solicitadas incluso por los mexicano-americanos. En lo que respecta a los partos, la situación es todavía más compleja, ya que una parte de las futuras madres mexicanas, a pesar de lo costoso $-\mathrm{y}$ esto no 
concierne solamente a la elite más rica-, no titubean en dar a luz en Estados Unidos para que sus hijos gocen de la ciudadanía.

Algunos años más tarde la interconexión alcanza al sistema educativo. A nivel superior, la proximidad geográfica refuerza las tendencias mexicanas en la continuación de estudios, en particular a nivel doctoral dentro de las universidades estadunidenses. El caso de Tucson es revelador, no sólo por el hecho de que los trecientos estudiantes mexicanos, allí presentes, constituyen una de las más importantes colonias de extranjeros, sino porque el número de estos estudiantes oscila muy claramente en función de la paridad de cambio entre el dólar y el peso (Cfr. Silver, 1991). De manera que el sector universitario se constituye muchas veces en pionero de la cooperación institucional entre las ciudades gemelas. La doble licenciatura en estudios intemacionales establecida conjuntamente entre la UABC y la Universidad Estatal de San Diego es un ejemplo, con un año en la Unidad de Tijuana, un año en San Diego, y un tercer año en una universidad del suroeste de Estados Unidos.

Poco a poco otros niveles de enseñanza se ven involucrados, no sólo debido a la importancia de la población hispana en el lado estadunidense, sino por la presencia creciente, en estas escuelas, de niños mexicanos venidos del otro lado, En una ciudad como Caléxico (cfr. Antonio Meza, 1989), se observa que de una poblacion de $56 \%$ de hispanos, $85 \%$ de los niños escolarizados son hispanoparlantes. Las encuestas han demostrado que el 50\% de estos niños residían en Mexicali y pertenecían a familias binacionales (uno de los padres, estadunidense). Ciertas encuestas subrayan la especificidad negativa en términos de potencial de aprendizaje de dicha población, incluso en relación con los "chicanos" en la medida que esos "niños-péndulo" llegan frecuentemente tarde debido a los embotellamientos que con mucha frecuencia se registran al cruzar la frontera; esto es por una parte, pero por otra parte, cabe señalar las dificultades de comunicación, que no sólo son lingüísticas, sino geográficas, entre los maestros y los padres de estos alumnos.

Lo más sorprendente es que también se da lo contrario; muchos niños de doble nacionalidad, hijos de padres residentes de Mexicali, asisten a las escuelas mexicanas. Las cosas se presentan como si todo sucediera en una ciudad normal desde el momento que las opciones y las necesidades interfieren con las "sectorizaciones" administrativas o lógicas en la escolarización de los niños. La banalización de la vida urbana de CaléxicoMexicali lo confirma a su manera: el desvanecimiento de la frontera, de hecho, transforma insensiblemente la doble ciudad en un conjunto único que obtiene de su misma heterogeneidad una cierta fuente de complementariedad y cohesion. 
Se trata de una red compleja, densa y variada, de relaciones cruzadas $e$ interacciones de lazos de reciprocidad y solidaridad que se desarrolla, realizando poco a poco, gracias a esas relaciones fronterizas vueltas transfronterizas, un espacio reticular en donde las ciudades no solamente constituyen nudos, sino campos bien precisos de aplicación. A tal punto esto, que esas construcciones en red terminan, por la multiplicidad de los flujos y fuerzas concentradas en las dos ciudades, por volver hacia las formas modulares de unidad del espacio urbano.

\section{LA FRONTERA AL INTERIOR DE LA CIUDAD}

\section{¿Hacia una continuidad territorial?}

La continuidad territorial es antes que todo la continuidad de la población. Si la americanización del lado mexicano es sobre todo económica, la hispanización - es mexicanización-creciente de las ciudades fronterizas estadunidenses es demografica y constituye un factor naturalmente decisivo en la gestación de esos nuevos territorios urbanos. La proporción de "hispanos" (concepto más amplio que el de población "de origen mexicano"), de por sí fuertes en los estados del suroeste ( $38 \%$ en Nuevo México, $26 \%$ en California y Texas, $13 \%$ en Colorado), crece hacia el sur: constituye $30 \%$ de la población de los condados fronterizos (si se excluye San Diego, es 50\%). Dicha población es mayoritaria en 16 de los 25 condados. La región metropolitana de Los Ángeles, con más de 2 millones de hispanos, incluye una comunidad cultural del tamaño de Guadalajara o Monterrey.

Una cuarta parte de la población de origen estrictamente mexicano, que cuenta con 13500000 personas (5.4\% de la población total) de los cuales $74 \%$ residen en California y Texas, se concentra en los condados fronterizos. Dicha concentración permite hablar de una cierta "reconquista silenciosa" de los territorios que en otra época fueron españoles y luego mexicanos.

En los mismos condados estadunidenses fronterizos, el diferencial de crecimiento de 1980 a 1990 , entre la población de origen mexicano $(+49.5 \%)$ y la población de otro origen $(+20.2 \%)$ confirma que el proceso de mexicanización prosigue su curso gracias a, por una parte, una fuerte fecundidad por parte de las madres mexicanas y, por otra, a la inmigración interna e internacional: en las zonas más agrícolas (Imperial, Yuma, Cameron) se da una especie de substitución de población, en el sentido de que los residentes de origen mexicano disminuye en cifras absolutas ( $\mathrm{Cfr}$. J. Revel-Muroz). 
La migración ilegal se orienta cada vez más hacia las ciudades (migrantes que eran urbanos en $35 \%$ en 1985 , y $90 \%$ hoy en día). Si por una parte, los porcentajes de la población "mexicana" disminuye desde los condados de Río Grande hasta los de Arizona y California (pasando de $90 \%$ a $50 \%$ y luego a menos de $20 \%$ ), por otra parte encontramos un número comparable, en números absolutos, de "mexicanos" en El Paso y San Diego.

Las formas de esta mexicanización, en la vivienda, los modos de vida y aun el "muralismo", por no citar un detalle, como la cocina y sobre todo el idioma y sus sincretismos diversos, no podrán desarrollarse dentro de este cuadro.

La continuidad territorial está marcada igualmente por la importancia de los intercambios en términos de energía, de agua o de riesgos en el medio ambiente que comienzan a actuar en la realidad - sobre todo en este último caso-, en la toma de conciencia de las solidaridades inevitables entre las dos vertientes urbanas de la frontera.

Esta globalidad de la problemática urbana se verifica en el campo de la contaminación. Si es atmosférica, ésta se burla de la frontera y de lo ancho del Río Grande. Las emanaciones que provienen de las fábricas de tratamiento minero diseminadas en el lado estadunidense, y presentes particularmente en El Paso, son consideradas como responsables de $20 \%$ de la contaminación ambiental -en lo que se refiere a gas sulfúrico-en América del Norte. El norte de México, y Ciudad Juárez principalmente, están fuertemente expuestos. Si es fluvial, ésta contamina muchas veces en sentido inverso. Es de esta manera que la ciudad de Nogales (Arizona), ubicada río abajo, recibe las aguas negras de Nogales (Sonora), su ciudad gemela. Lo mismo pasa en San Diego, en donde desemboca el Río Tijuana, siendo que tres cuartas partes de su lecho se encuentra en Tijuana. En este último caso, la solidaridad se encuentra reforzada con la multiplicidad de los intercambios de daños o riesgos en la medida que las corrientes marinas de la bahía, tenderían en sentido contrario a propagar la contaminación californiana hacia la ciudad mexicana, en tanto que sobre el plano atmosférico, la inversión diurna de las brisas dominantes envía alternativamente los efluvios de la ciudad estadunidense hacia Tijuana y los bedores de la ciudad mexicana bacia San Diego.

La explosión urbana en los dos lados de la frontera, ha sido en primer lugar, paralela, independiente y desfasada. Con la intensificacion de los fenómenos migratorios, ésta ha evolucionado hacia formas graduales de interdependencia. El proceso actualmente en marcha, prolonga, profundiza y acelera esta tendencia orientándose hacia la integración transfronteriza. 
Cuadro 7. Crecimiento de la población fronteriza de condados estadunidenses y municipios mexicanos. Crecimiento de la población de origen mexicano (millones).

\begin{tabular}{|c|c|c|c|c|c|c|c|c|c|c|}
\hline $\begin{array}{l}\text { Principales } \\
\text { condados }\end{array}$ & $\begin{array}{l}\text { A* }^{*} \\
\text { Pop } \\
1990\end{array}$ & $\begin{array}{c}\text { A } \\
\% \\
1990\end{array}$ & $\begin{array}{c}\text { A } \\
\text { Var } \\
80 / 90\end{array}$ & $\begin{array}{c}\text { B* } \\
\text { Origen } \\
\text { Mex. }\end{array}$ & $\begin{array}{c}\mathrm{B} / \mathrm{A} \\
\%\end{array}$ & $\begin{array}{c}\text { B } \\
\text { var \% } \\
80 / 90\end{array}$ & $\begin{array}{l}\text { Principales } \\
\text { municipios }\end{array}$ & $\begin{array}{l}\text { Pop* } \\
1990\end{array}$ & $\begin{array}{c}\% \\
1990\end{array}$ & $\begin{array}{l}\text { Var } \\
80 / 90\end{array}$ \\
\hline San Diego & 2498 & 48.1 & 34.2 & 439 & 17.6 & 92.5 & Tijuana & 743 & 19.5 & 61.0 \\
\hline Imperial & 109 & 2.1 & 18.7 & 69 & 63.1 & 39.3 & Mexicali & 602 & 15.8 & 18.0 \\
\hline Yuma & 107 & 2.1 & 18.0 & 41 & 38.7 & 66.7 & San Luis R & 112 & 2.9 & 20.2 \\
\hline Pima & 669 & 12.9 & 25.9 & 148 & 22.1 & 47.0 & Nogales & 107 & 2.8 & 57.4 \\
\hline Doña Ana & 136 & 2.6 & 45.2 & 69 & 50.9 & 62.6 & & & & \\
\hline El Paso & 592 & 11.4 & 23.3 & 392 & 66.2 & 39.0 & Juárez & 798 & 20.9 & 40.6 \\
\hline Webb & 133 & 2.6 & 34.2 & 119 & 89.3 & 37.5 & N. Laredo & 218 & 5.7 & 7.2 \\
\hline Hidalgo & 384 & 7.4 & 35.4 & 311 & 81.2 & 40.3 & Reynosa & 283 & 7.4 & 33.2 \\
\hline Cameron & 260 & 5.0 & 24.0 & 201 & 77.2 & 45.0 & Matamoros & 303 & 8.0 & 27.0 \\
\hline Subtotal & 4885 & 93.4 & 30.5 & 1789 & 36.6 & 52.4 & Subtotal & 3164 & 83.0 & 34.4 \\
\hline Total frontera & 5197 & 100 & 29.6 & 1974 & 38.0 & 49.5 & Total frontera & 3810 & 100 & 31.0 \\
\hline
\end{tabular}

Fuente: Censos de población de los Estados Unidos 1980,1990 y Censo General de Población y Vivienda, México, 1980,1990.

* miles de personas

Cuadro adaptado de Roberto Ham Chande "La participación de la población de origen mexicano en el crecimiento demográfico de la frontera entre México y Estados Unidos". CoLEF. Ponencia presentada en el Congreso de.Población de Veracruz, abril de 1992. 
Tres observaciones podrian simbolizar dicha progresion, ese paso que sería de la fisión de las ciudades gemelas, hacia la fusión:

1) El continuo tráfico de trabajadores mexicanos a través del Río Grande, entre Ciudad Juárez y el Paso, teóricamente clandestinos, pero de hecho trivial;

La experiencia ha mostrado que ya no se trata sólamente de "braceros", sino también - y sobre todo- de "commuters" ilegales que cruzan cotidianamente el río, $\mathrm{y}$ aun "commuters" legales que prefieren esta forma de paso ipara ganar tiempo en relación al Puente Internacional!

2)La multiplicidad de los viajes de regreso temporal de los hispanos, con estrategias de compra a ambos lados, según los productos, trivializa una vez más la frontera; $y$

3)La residencia de numerosos mexico-americanos en la ciudad mexicana. Esto se puede constatar por las mañanas, en las garitas de Tijuana o de Mexicali, con las largas filas de autos - en su mayoría con placas californianas - que cruzan la frontera en dirección a Estados Unidos. Se trata pues de una elección para vivir en el lado mexicano, y no de una obligación. Las estrategias de vivienda se han vuelto también transnacionales y funcionan en los dos sentidos. A mediano plazo, podriamos preguntamos en qué medida las zonas residenciales litorales, situadas entre Tijuana y Ensenada, destinadas a la clientela estadunidense, no van a evolucionar hacía viviendas semidefinitivas de pensionados $\mathbf{y}$, por lo menos en la parte septentrional, hacia residencias principales, dada la diferencia de precio para los commuters.

\section{¿TIJUANA-SAN DIEGO, UNA NUEVA FRONTERA PARA LA METRÓPOLI TRANSFRONTERIZA?}

"San Diego think of itself a border town, but, in reality, it is part of the functionning metropolitan region of San Diego-Tijuana"*

(Herzog, 1990:208).

\section{Una ciudad, dos ciudades}

La densificación y la sistematización de los intercambios transfronterizos se traducen poco a poco en la organización del espacio urbano. Las primeras formas son antiguas. Éstas, aunque reducidas, pero eso sí bien concretas, se expresan principalmente en el barrio "internacional" de

* N. del E."San Diego cree que es una ciudad fronteriza por sí misma, pero en realidad es parte de la región metropolitana de San Diego-Tijuana". 
Tijuana, estirado entre la aduana con sus múltiples tiendas de curiosidades y la avenida principal (Revolución), en donde se concentra la casi totalidad de los turistas. La americanización del lado mexicano parece inscribirse también en el barrio del Río, recientemente construido en detrimento de la vieja ciudad de Tijuana y sobre todo en el lugar de Cartolandia, una inmensa ciudad perdida suprimida hace veinte años. Las grandes avenidas, los edificios, los hoteles, los "malls" de los centros comerciales y las dos torres de la Plaza le imponen formas nuevas a la ciudad. Aunque éstas evocan más la modernidad o la americanización que lo fronterizo.

Por el contrario, la huella mexicana se ve más fuerte en la arquitectura de San Diego. Muchas quintas de La Jolla, así como tiendas y edificios antiguos, como la estación del ferrocarril (Santa Fe Railroad Depot), o recientes como la Plaza Center, muestran materiales o formas típicamente hispanas. En realidad éstas no traducen ni una herencia anterior a 1848 , ni una mexicanización reciente, expresan sencillamente un estilo "californiano", nacido de una preferencia arquitectónica por lo exótico-original, y esto gracias a referencias tomadas de influencias propias del modelo anglo del Este. Se trata pues de algo nada impuesto ni mucho menos buscado como valor historico.

Por el contrario, se podrá observar que en los barrios latinos, en donde las apariencias de la pobreza (falta de mantenimiento, principalmente) resaltan más como diferencia que como marcas de hispanidad. El "barrio Logan" al sur del puerto, podría ser un ejemplo, aunque un cambio reciente podria indicar la irrupción, también reciente, de murales, principalmente en los pilares del viaducto Coronado, y esto de acuerdo con la gran tradición del arte mexicano en custión de frescos murales.

Esta evolución se debe en adelante a la afirmación de la diferencia y a la voluntad de los chicanos por mostrar - en términos de su modelo urbano- su oposición a la hegemonía anglo. Se sabe que la afirmación étnica de una minoría y sus estrategias territoriales dependen de las relaciones de fuerza con la sociedad dominante. En el caso de San Diego, el crecimiento demográfico de la población hispana constituye un fenómeno reciente que, para el gran pesar de ciertos medios anglos, modifica esa relación de fuerza, colocando a la ciudad tradicional entre dos frentes "hispánicos": el de la frontera y el de los chicanos.

\section{Una ciudad, dos ciudades o tres}

A partir de dos plazas-fuertes territoriales, constituidas por los gethos latinos del barrio Logan por una parte y el de San Isidro, pegado a la frontera, por otra parte, se opera una cierta mexicanización de los barrios 
Cuadro 8. Crecimiento de la población hispana en el condado de San Diego, 1960-1990.

\begin{tabular}{lrrrrrrr}
\hline Población & $\begin{array}{r}1960 \\
\text { Total }\end{array}$ & $\begin{array}{r}1970 \\
\text { Total }\end{array}$ & \multicolumn{1}{c}{$\begin{array}{c}1980 \\
\text { Total }\end{array}$} & $\begin{array}{c}1990 \\
\text { Total }\end{array}$ & $\begin{array}{c}\text { Incremento } \\
1960-1990\end{array}$ & $\begin{array}{r}\text { Proporción } \\
\text { en 1990\% }\end{array}$ \\
\hline Total & 1033011 & 1357854 & 1861846 & 2498016 & 1465005 & 141,8 & 100 \\
Hispanos & 64810 & 174209 & 275177 & 510781 & 445971 & 688,1 & 20,45 \\
Otros & 968201 & 1183645 & 1586669 & 1987235 & 1019034 & 105,3 & 79,55 \\
\hline
\end{tabular}

Fuente: Censos federales de 1960,1970,1980 y 1990; San Diego Economic Bulletin, vol 39, núm. 7, julio 1991.

sur y este de San Diego. Dicho proceso avanza gracias al espacio que va dejando el tropismo espontáneo con el que la ciudad anglo se mueve en dirección del mar y hacia el norte, y de la proximidad de los empleos industriales ligados a la frontera. El fenómeno se mantiene y se amplifica naturalmente, a la vez como efecto consecuente al rechazo resentido en relación con los anglos, y por el mismo juego de afiliaciones de proximidad que se van creando.

Las diferentes cartografías de los residentes mexicano-americanos, de los lugares en los que compran los mexicanos, de los lugares en los que trabajan los commuters, revelan esa mexicanización progresiva del sur de San Diego y esa tendencia al coligamiento que se observa en los núcleos primitivos de Logan y San Isidro, paralelamente a la transformación progresiva de San Diego en verdadera ciudad fronteriza.

El fenómeno de metropolización transfronteriza, "transborder metropolis" (cfr. L. Herzog, 1990) se complementa con un guión espacial, con la constitución de un San Diego-sur, como espacio urbano de transición entre el San Diego californiano y Tijuana. En Tijuana, inversamente, ciertos barrios del norte forman a su vez una zona de transición americanizada: "In some ways, $I 94$, the interstate freeway running from east to west toward downtown San Diego serves more to divide hispanic or non hispanic San Diego, than does the internacional boundary" (cfr. L. Herzog, 1990:186).*

\footnotetext{
* N. del E. De cierta manera, la carretera I 94, la autopista interestatal que corre de este a oeste hacia el centro de San Diego, sirve más para dividir a los hispanos de los no hispanos de San Diego, que la propia línea internacional.
} 
En una cierta medida, la urbanización lineal a lo largo de la costa de Baja Califomia, entre Tijuana y Ensenada, con los fraccionamientos turísticos, se inscribe también como una prolongación de este mecanismo, al crear un simétrico meridional y más popular en las zonas residenciales balnearias del norte de San Diego.

El desarrollo de los terrenos de golf y de los proyectos de marinas en esta zona, dan fe de la función potencial de reserva de espacio que éstas pueden jugar para la promoción de bienes y raíces. Desde este punto de vista, los terrenos de golf constituyen un factor atractivo primordial dentro del nuevo modelo urbano ecolo-deportivo de California, al mismo título que la diferencia de precios (el condado de San Diego cuenta con 70 terrenos de golf).

Es, finalmente, a la escala de una megalópolis Los Ángeles-San DiegoTijuana-Ensenada, a lo largo de 300 kilómetros, que se sitúan las salidas de inversiones, equipo y acondicionamiento urbano transfronterizo. La Baja California mexicana, de Tijuana a Ensenada, que dispone de espacio, se ha convertido en una salida para la infraestructura de transporte internacional al servicio de la gran California.

Frente a la congestión del aeropuerto internacional de San Diego, las autoridades locales de este condado le han propuesto a México la creación de un aeropuerto binacional, tomando como modelo el de Ginebra o de Basilea, para que reuniera en una misma unidad de operación una antigua pista militar estadunidense y el actual aeropuerto de Tijuana, localizado a lo largo de la frontera, en el corazón de la zona en donde se concentran las maquiladoras. Hasta ahora las autoridades mexicanas federales no han consentido lo que ellos consideran como un proyecto que cuestionaría la soberanía del país, prefieren mejor hablar de aeropuerto fronterizo paralelo.

Por el contrario, el proyecto de modernización del puerto de Ensenada, como complemento del de Los Ángeles, es considerado favorablemente a ambos lados de la frontera, con lo cual se espera dinamizar la economía regional,

A partir del momento en que toda la evolución va en el sentido de una disminución a más o menos largo plazo de los últimos obstáculos materiales, institucionales o psicológicos, lo ordinario de la frontera deberia confirmar y desarrollar esos procesos generalizados, a la vez modularios y sistémicos, de metropolización transfronteriza.

Una evolución y un reto cuya originalidad e interés, a la vez humana, científica y política, sugiere un alcance que va mucho más allá del cuadro local. 


\section{CONCLUSIóN}

A su escala local, la evolución urbana, de la biurbanización hacia la conurbación, aparece como emblemática y anunciadora de una evolución general de integración mexicano-americana en el seno de un conjunto regional transfronterizo (mexamérica), y quizas contintenal (TLC) en gestación.

Después de haber vivido frente a frente, luego lado a lado, las ciudades fronterizas aprenden ahora a vivir juntas. Las antiguas parejas fronterizas evolucionan de la coexistencia pacífica a la complexidad activa, a partir de mecanismos complejos de interacción que transforman los espacios modulares, antiguos, diferenciados e independientes en espacios recticulares cada vez más interpenetrados y funcionales. Aunque el problema de las relaciones futuras en las conurbaciones fronterizas, en términos de poder y de imagen, se remite una vez más a las relaciones ambiguas de atracción-repulsion, solidaridad-afirmación de identidad, que marcan de manera aún más general la relación México-Estados Unidos. Asfi, dentro de la conurbación en donde El Paso se terciariza mientras que Ciudad Juárez se industrializa con las maquiladoras,

¿será El Paso el cerebro y Ciudad Juárez el cuerpo de ese monstruo bicéfalo que no acaba de crecer? ¿Aceptará México esta nueva forma de dominación, aún cuando la población de Juárez sea hoy en día casi tres veces más importante que su gemela? (cfr. Flosse, 1989).

Esta evolución no avanza pues sin reticencias, debilidades y retrasos, igualmente sensibles a nivel regional.

El intercambio dialogístico desemboca, de hecho, rara vez en acuerdos formales. La convergencia conduce excepcionalmente a la cooperacion. Los mecanismos naturales o espontáneos, las fuerzas del mercado, el poder de la necesidad, y cada vez más, las alianzas familiares, inducen hacia una comunidad de intereses y una solidaridad, de hecho, poco abordada institucionalmente.

Todos estos ejemplos anotados acerca de Tijuana-San Diego (cfr. Mesa de Otay y el proyecto de un aeropuerto internacional), no son más que la excepción que confirma la regla, que es la de la separación - cuando no es el de la ignorancia recíproca- de los poderes municipales, y de la ausencia de organismos, de status o de función metropolitana, ya sea sobre el plano de la gestión, de la planificación o del diagnóstico. Una ilustración típica de este desfase entre las formas del mercado y el espacio político se ve en el hecho mismo de que, hacía unos cuantos años, era prácticamente imposible encontrar mapas que cubrieran los dos lados de 
las parejas urbanas, Los mapas estadunidenses se terminaban en el sur, en la frontera misma, con un espacio en blanco, simétrico al que los documentos mexicanos dejaban en la parte norte.

Las costumbres, los intereses políticos electorales, las rivalidades de poder, se oponen - aun cuando no fuese sólo en la frontera-, a toda delegación de soberanía y a cualquier riesgo de perder el poder por parte de las élites políticas que reinan en los diferentes niveles administrativos, igualmente en lo que respecta a los funcionarios nacionales o locales, celosos de sus prerrogativas.

A todo esto, viene y se agrega un doble desfase, específico a la frontera. El primero se refiere a los grados de democratizacion, se trata de un retraso considerable del lado mexicano en lo que respecta a la calidad de la democracia local y particularmente municipal. El segundo se refiere a las diferencias de competencias en el manejo de los asuntos urbanos, debido principalmente a las diferencias en cuestiones de jurisdicción (condados en Estados Unidos), pero sobre todo de financiamiento de las entidades territoriales correspondientes a los dos países. En este sentido, al lado mexicano le corresponde hacer un esfuerzo considerable para lograr una cogestion relativamente equilibrada: el puro presupuesto de "transporte" de San Diego equivale a 4 veces el presupuesto total de Tijuana.

Si se toman en cuenta los problemas de las ciudades estadunidenses, particularmente en lo que se refiere a agua, al espacio, al medio ambiente y a la migración, los Estados Unidos resultan demandantes de cooperación y de "crossborder planning".

Las ciudades gemelas deben tomar conciencia, a nivel urbano, de que el desarrollo de la cooperación y de la integración debe pasar a través de ciertas formas de redistribucion de medios y recursos, a la manera como lo hacen las metrópolis clásicas entre centro y suburbio, en beneficio de las zonas pobres, en este caso mexicanas.

Es de imaginarse la complejidad del problema y las incógnitas cronológicas que gravitan en torno a su resolución, aun cuando ésta pueda ser ineluctable, como lo subraya L. Herzog: "Boundary cities have become so functionnally interwined that their futures are inextrically bound, whether or not the two national government are able to devise formal procedures in addressing border-related problems".*

\footnotetext{
* N. del E. "Las ciudades fronterizas han pasado a estar tan entrelazadas funcionalmente, que sus futuros están inextricablemente ligados, ya sea que los dos gobiernos nacionales sean capaces o no de idear procedimientos formales para dar tratamiento a los problemas referentes a la frontera".
} 
En fin, por ahora se plantea la pregunta, en el plano científico, acerca del grado real de integración que han alcanzado los sistemas urbanos transfronterizos.

Considerada la desventaja que resulta de la escasa cooperación a nivel oficial, pero, inversamente, considerada la importancia de las interacciones y de las traducciones espaciales observadas ¿habrá esta frontera superado los niveles de integración que han alcanzado ciertas ciudades de Europa, como por ejemplo entre Lille-Roubaix-Tourcoing (Francia) y Courtrai (Bélgica), entre Bâle (Suiza) y Mulhouse (Francia), o entre Ginebra (Suiza) y Annemasse (Francia)? La respuesta exige sin lugar a dudas un estudio comparativo bien preciso.

Tenemos la impresión de que, en el caso de Ginebra-Annemasse, el proceso se encuentra todavía en el nivel de yuxtaposición de las dos ciudades fronterizas, más que en el de la configuración de una metrópoli; es probable, sin embargo, ¿a una escala más pequeña, se podría buscar en la zona trinacional de Longwy (Luxemburgo, Alemania, Francia) y su proyecto de eurociudad, una cierta analogía con los avances de Tijuana/San Diego o de "Paso del Norte"?

\section{BIBLIOGRAFÍA}

ACUÑA, Rodolfo. 1988. Occupied America, Harper Collins, Nueva York (EUA).

ALEGRÍA, Tito. 1989. "Ciudad y procesos transfronterizos", Frontera Norte, núm. 2, julio-diciembre. Colegio de la Frontera, Tijuana (México).

- 1990. "Ciudad y transmigración". Frontera Norte, núm. 4, Vol. 2, julio-diciembre. Colegio de la Frontera, Tijuana (México).

BARRERA Herrera, Eduardo. 1992. "De carreteras electrónicas a ciudades cableadas". En: Ciudades. Dinámica urbana regional a los 90 ', núm. 13, Janvier. México, D.F.

BECKER, Angélica. 1990. "People in Business". En: Colin Flaherty, Mexico Business News, vol. 1, núm. 1. Janvier.

BIRDSALL, Stephen S. 1992. Regional Landscapes of USA and Canada, John Wiley, Nueva York.

CASTILLO, Víctor. 1992. Economía fronteriza y desarrollo regional, UABC, Mexicali.

ESTRELLA, Gabriel. 1992. "The Floating Population of the Border", En Demographic Dynamics of the U.S.-Mexico Border, Weeks John R. y Ham-Chande Roberto (ed.), Texas Western Press, El Paso. 
FERNÁNDEZ, Raúl, A. 1989. The Mexican American Boder Region, University of Notre Dame, Indiana.

FLOSSE, Geneviève. 1989. "EPJAZ Coopération dans un désert. Réseaux de services urbains et coopération transfrontalière Etats-UnisMexique". LATTS. Ecole nationale des Ponts et Chaussées, Université de Paris XII, Paris.

HERZOG, Lawrence, A. 1990. When North meets South. Center for Mexican American Studies. Austin University Press.

MEZA Estrada, Antonio, 1989. "Educación y Migración en la Frontera Caléxico-Mexicali". Estudios Fronterizos, vol. 8, núm. 18/19, eneroabril/mayo-agosto. Mexicali, México.

PAVLAKOVIC, Vera. y Hak-Hoon Kim. s/f."Outshopping by Maquilas Employees", Arizona Review. A. Silvers. Ed. University of Arizona. Tucson.

REVEL-MOUROZ, Jean. 1993. "La nouvelle Frontière au coeur de l'intégration aux Etats-Unis" dans "Le Mexique à l'Aube du Troisiéme Millénaire", Marie-France Prévot Schapira y Jean Revel-Mouroz, (coord.), Editions de l'IHEAL, Paris.

RUIZ, Olivia. 1992. "Visitando la matria ...", Frontera Norte, vol 4, núm. 7, enero-junio. El Colegio de la Frontera, Tijuana (México).

SILVERS, A.L. 1991. Tucson and Mexico Current Economic Links and the F.T.A. Office of economic Development. International Program. Ciudad de Tucson. (EUA).

VANNEPH, Alain, y Jean Revel-Mouroz. 1989. "Ciudad Juárez-El Paso: le boom des maquiladoras", CREDAL/America Communication Film Video 8. 40'1989. 